\title{
The Impact of Fiber Core Ellipticity and Modal Coherency on Few Moded Erbium Doped Fiber Amplifiers
}

\author{
Ee-Leong Lim ${ }^{(1)}$, Sonali Dasgupta, Qiongyue Kang, Jae M. O. Daniel, \\ Francesco Poletti, Shaif-ul Alam and David J. Richardson
}

(1) Optoelectronics Research Centre, University of Southampton, Southampton, Hampshire, SO17 1BJ, United Kingdom, ell@orc.soton.ac.uk

\begin{abstract}
We numerically demonstrate that the few moded erbium doped fiber amplifier models based on linearly polarized and vector modes are equivalent when the fiber core exhibits slight ellipticity and the signals in the guided modes are incoherent. These conditions are fulfilled in a practical mode division multiplexed system.
\end{abstract}

\section{Introduction}

Mode division multiplexed (MDM) optical communication systems are currently under intense investigation as a means to overcome the capacity limit of single-mode systems ${ }^{1}$. To realize the energy and cost savings offered by MDM systems, the individual guided modes should be simultaneously amplified within a few moded erbium doped fiber amplifier (FM-EDFA). An accurate FM-EDFA model is essential to develop a fundamental understanding and to reliably design FM-EDFA devices with suitable operational performance. Ideally, a FM-EDFA should exhibit a low mode dependent gain (MDG) in order to lower the system outage probability $^{2}$. Most numerical results reported so far are based on use of a scalar linearly polarized modes (LP-modes) model ${ }^{1}$. However, the non-LP $0 \mathrm{~m}$ mode groups are composed of four vector modes (the real modes of the system) that exhibit very close yet different phase velocities ${ }^{3}$. Recently, it has been shown that simulation results based on a full vector mode model (VM) can differ significantly from those based on an LP-mode model (LM) ${ }^{3,4}$. However it is also well known that in fibers with an elliptic core, the non- $\mathrm{HE}_{1 \mathrm{~m}}$ modes evolve into linearly polarized vector modes (LP-VMs) due to birefringence ${ }^{5,6}$.

In this paper, we show the conditions that lead to the equivalence of LM and VM. First, the evolution of the $\mathrm{HE}_{21}$ and $\mathrm{TE}_{01}$ modes into linearly polarized vector modes (LP-VMs) is presented. Next, we discuss the interference of partially coherent signal. Finally, we study the modal power evolution in a FM-EDFA.

\section{Modes in fiber with slight core ellipticity}

A step-index fiber with a core/cladding refractive index of $1.454 / 1.45$ is studied. The fiber core ellipticity is quantified by the percentage difference between the major and the minor axes, denoted as the deviation $(D)$. The minor axis is parallel to the horizontal axis and is fixed at $8 \mu \mathrm{m}$. Thus, a $D$ of $0.5 \%$ corresponds to a fiber core with minor and major axes of $8.00 \mu \mathrm{m}$ and $8.04 \mu \mathrm{m}$ respectively.

The effects of form and stress induced birefringence are modeled using COMSOL Multiphysics $4.3 \AA$, using the following parameters for silica ${ }^{6}$ : thermal expansion coefficient of cladding/core of $1 \times 10^{-6} / 2 \times 10^{-6} \mathrm{~K}^{-1}$, Young's modulus of $78 \mathrm{GPa}$, Poisson's ratio of 0.42 , density of $110.3 \mathrm{~kg} / \mathrm{m}^{3}$, first/second stress optical coefficient of $0.75 \times 10^{-12}$ and $4.2 \times 10^{-}$ ${ }^{12} \mathrm{~m}^{2} / \mathrm{N}$, reference temperature of $1273.2 \mathrm{~K}$ and operating temperature of $293.15 \mathrm{~K}$.

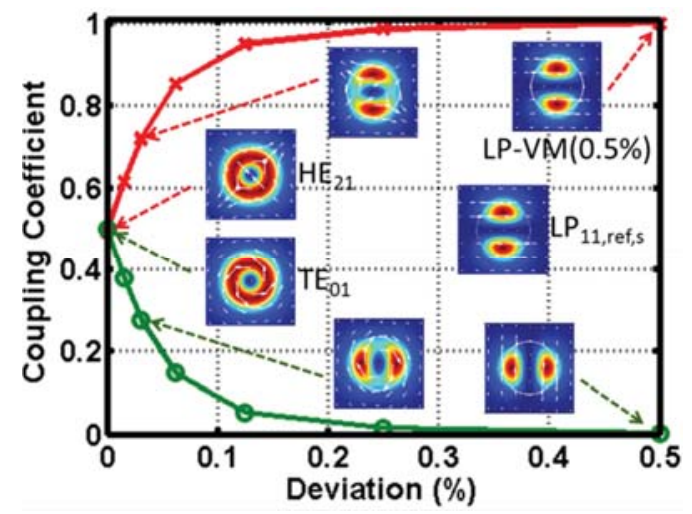

Fig. 1: The coupling coefficient of the $L P_{11, \text { ref,s }}$ into the two vector modes at different deviations $(D s)$. The insets show the mode intensity profiles (MIPs) of the vector modes at various $D$ s and of the $L P_{11, \text { ref,s. The }}$ white circles outline $8 \mu \mathrm{m}$ radius circles. The white arrows illustrate electric fields.

A reference $L P_{11}$ mode $\left(L P_{11, \text { ref,s }}\right)$ is first constructed from the vectorial sum of equal signal powers in the $\mathrm{TE}_{01}$ and $\mathrm{HE}_{21}$ modes of the fiber at $D=0.0 \%$. The mode intensity profile (MIP) of $L P_{11, \text { ref,s }}$ is shown inset in Fig.1. Next, this $L P_{11, \text { ref,s }}$ is used as the excitation field at the fiber input. The $L P_{11, \text { ref,s }}$ couples only to two guided modes with the coupling coefficient $(\eta)$ at various values of $D$ shown in Fig. 1. At $D=0.5 \%$, the $L P_{11 \text {,ref,s }}$ effectively couples to only one of the LP-VMs, i.e. $\eta \approx 1$. This mode with $\eta \approx 1$ at $D=0.5 \%$ will subsequently be denoted as LP$\operatorname{VM}(0.5 \%)$. Due to the choice of the direction of the minor axis, the maxima of the LP-VMs 
coincide with the maxima of $L P_{11, \text { ref,s }}$. A different orientation of the excitation field with respect to the LP-VMs will clearly result in a different coupling condition.

\section{Modal coherency in MDM system}

With the assumption that the power guided in the fiber modes is stationary, the resultant average modal intensity (RA-MI) $\left(I_{R}(r, \phi, z)\right)$ for the combination of partially coherent light guided in the vector modes is given by ${ }^{7}$ :

$$
\begin{aligned}
I_{R}(r, \phi, z)= & \operatorname{Re}\left[\sum_{m}^{K} \sum_{n}^{K} \sqrt{I_{m}}{\sqrt{I_{n}}}^{*}\left|\gamma_{m n}\right|\right. \\
& \times \exp \left(-j\left(\beta_{m}-\beta_{n}\right) z+j \arg \left(\gamma_{m n}\right)\right) \\
& \left.\times E_{m}(r, \phi) E_{n}^{*}(r, \phi)\right]
\end{aligned}
$$

where $m$ and $n$ are the indices of the vector mode, $K$ is the total number of modes, $\beta_{m}$ is the propagation constant and $E_{m}(r, \phi)$ is the normalized field distribution and $z$ is the propagation distance. The amplitude of the electric field $A_{m}$ of $m^{\text {th }}$ mode is a random function of time and is related to the average intensity through:

$$
I_{m}=\left\langle\left|A_{m}\right|^{2}\right\rangle
$$

where the symbol $\langle\bullet\rangle$ denotes an ensemble average over many realization of the random function. $\gamma_{n m}$ is the complex degree of coherence between the $m^{\text {th }}$ and $n^{\text {th }}$ modes and is defined as:

$$
\gamma_{m n}=\frac{\left\langle A_{m} A_{n}^{*}\right\rangle}{\sqrt{I_{m}} \sqrt{I_{n}}} .
$$

It is a measure of the degree of coherence between the signal guided in the two modes ${ }^{7}$.

There are two important limits for Eq. (1). On one hand, when all modes are coherent, $\left|\gamma_{n m}\right|=1$ for all $m$ and $n$. The Eq. (1) becomes the typical formula for the vectorial combination of coherent vector modes with phase difference of $\arg \left(\gamma_{\mathrm{nm}}\right)$ between the $m^{\text {th }}$ and $n^{\text {th }}$ modes. On the other hand, when the modes are incoherent, $\left|\gamma_{n m}\right|=1$ only when $n=m$ and $\left|\gamma_{n m}\right|=0$ otherwise. The Eq. (1) then becomes the summation of the optical intensities of modes and the phase relationship between the different modes is random. If $0<\left|\gamma_{n m}\right|<1$, the modes are said to be partially coherent.

To illustrate the impact of $\gamma_{n m}$ on RA-MI, the $\mathrm{LP}-\mathrm{VM}(0.5 \%)$ is combined with an equal power of co-polarized $\mathrm{HE}_{11}$ mode (see Fig. 2(a)-(b)) with $\arg \left(\gamma_{n m}\right)=0$. The RA-Mls at 3 different values of $\gamma_{n m}$ are shown in Fig. 2 (d)-(e). When the two modes are coherent, modal beating results in a strong interference effect at the two lobes of the LP-VM(0.5\%) (see Fig. 2(d)). When the mode becomes incoherent, the RA-MI becomes the summation of their MIPs. It should be noted that if the $\mathrm{HE}_{11}$ and $\operatorname{LP}-\mathrm{VM}(0.5 \%)$ are cross- polarized, the two modes will not interfere and the RA-MI also corresponds to the summation of their MIPs.

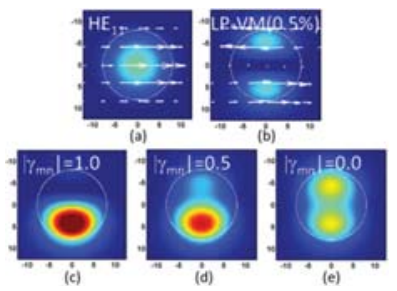

Fig. 2: (a)-(b): The MIPs of $\mathrm{HE}_{11}$ mode and LP-VM $(0.5 \%)$. The white arrows illustrate the electric field. (c)-(e): The resultant average modal intensity of the combination of equal power of $\mathrm{HE}_{11}$ mode and LPVM at 3 different values of $\left|\gamma_{\mathrm{rm}}\right|$ and $\arg \left(\gamma_{\mathrm{rm}}\right)=0$.

In a high bit rate phase-modulated MDM system, there is no continuous wave (cw) power at the carrier frequency for PSK, QPSK and QAM modulation schemes. Hence, there is no interference effect due to the $\mathrm{cw}$ power component. Furthermore, in an MDM system, the different signal modes act as independent information channels. Hence, the digital data and phase modulation on the different signal modes is independent (In fact, in the current MDM transmission experiments, the signal modes are intentionally symbol delayed for decorrelation $^{8}$ ). This independent phase modulation results in a random phase variation among the signal modes. Thus, the signal modes in MDM system can be in general considered as incoherent modes.

\section{Few moded fiber amplifier}

With the resultant signal and pump RA-Mls given by Eq. (1), the population inversion and the modal power evolutions (MPEs) of signal and pump modes can be simulated based on the VM described in ref. 3-4. We study a codirectional pumped $4 \mathrm{~m}$ long FM-EDFA. The FMEDFA has a uniform erbium ion doping profile with a doping radius of $8 \mu \mathrm{m}$ and a doping concentration of $15 \times 10^{24} \mathrm{ion} / \mathrm{m}^{3}$. The input signal mode at $1550 \mathrm{~nm}$ are $1 \mathrm{~mW}$ of co-polarized $L P_{01} / H E_{11}$ and $L P_{11, \text { ref,s }}$ modes respectively. The $\eta$ of $L P_{11, \text { ref, }}$ into LP-VMs are those shown in Fig. 1. The pump mode at $980 \mathrm{~nm}$ is $250 \mathrm{~mW}$ of reference $L P_{11}$ pump mode $\left(L P_{11, \text { ref, }}\right)$, which is constructed in a similar manner as the $L_{11, \text { ref,s. }}$. Since the pump mode also exhibits a similar type of $D$ dependency as the signal mode, the $\eta$ of $L_{11, \text { ref,p }}$ into the pump LP-VM has also been calculated and incorporated in the simulation. The fiber core ellipticity is assumed to be the same across the fiber length. The $\arg \left(\gamma_{n m}\right)$ is set to 0 for all pump and signal modes. Other simulation parameters can be found in ref. 3 .

Fig. 3(a) shows the signal MPEs based on the VM at different values of $D$ with coherent 
modes. The dashed lines show the sum of MPEs of the two LP-VMs excited by LP ${ }_{11, \text { ref,s }}$ and we denote them as $L_{11}$. At $D=0.00 \%$ (red), the MPE of $L P_{01}$ is higher than that of $L P_{11}$. However, at $D=0.50 \%$ (green), the MPE of LP 11 is higher than $\mathrm{LP}_{01}$. Hence, a change in $D$ can drastically alter the behavior of a FM-EDFA. Fig. 3(b) compares the behavior based on VM with $D=0.50 \%$ (green) with coherent modes, and that simulated based on LM at $D=0.00 \%$ (purple). The MPEs from LM and VM are different due to the modal beating between the two signal modes, i.e. the LP-VM(0.5\%) and the $\mathrm{LP}_{01}$.

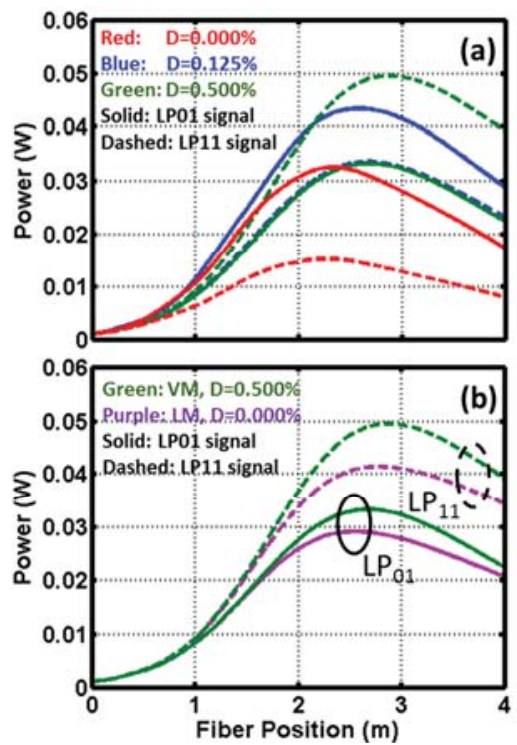

Fig. 3: (a) The MPEs based on the VM at various values of $D$ with coherent modes. (b) Comparison of the MPEs based on the VM at $D=0.50 \%$ with coherent modes and based on LM at $D=0.00 \%$.

To illustrate this, the input signals are changed to $1 \mathrm{~mW}$ of $\mathrm{LP}_{01}$ and $1 \mathrm{~mW}$ of $\mathrm{LP}$ $\mathrm{VM}(0.5 \%)$ and re-simulated under 3 cases: (i) VM with coherent modes, (ii) VM for incoherent modes and (iii) LM. Fig. 4(a) shows the MPEs for case (i) and (ii) which exhibit the same trend as Fig. 3(b). Fig. 4(b) shows the normalized population inversion $\left(N_{2}\right)$ at 3 spatial positions (A, B and C). The rapid fluctuation of $N_{2}$ at $A$ in case (i) is due to the modal beating between the two signal modes ${ }^{3}$. In case (ii), there is no rapid fluctuation in the $N_{2}$ at A. (Note that the $N_{2}$ at B and $C$ in the case of (i) and (ii) overlap well with each other and are therefore difficult to discern.) Meanwhile, the results from case (ii) and (iii) are exactly the same. In fact, the equations for LM can be derived from (1)-(3) by assuming incoherent modes, i.e. the LM becomes equivalent to the VM.

We note that the above conclusion is based on the orientation of $\operatorname{LP}-\mathrm{VM}(0.5 \%)$ and $\mathrm{LP}_{11, \text { ref,s }}$ shown in Fig.1, i.e. they are co-polarized. Meanwhile, it can also be shown that LM and
VM in the case of cross-polarized mode are also equivalent because they do not interfere. Hence, independent of the spatial and polarization directions, the LM and VM are equivalent when the fiber core exhibits a slight ellipticity and the signals in the guided modes are incoherent.
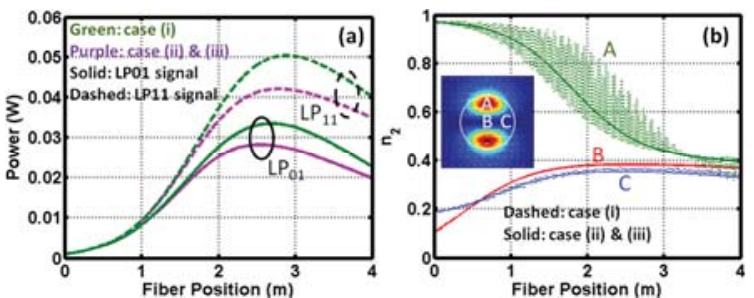

Fig. 4: (a) The MPEs for different cases (b) The $N_{2}$ at 3 transverse spatial locations shown in the inset, i.e. green:A $(0 \mu \mathrm{m}, 5 \mu \mathrm{m})$, red:B $(0 \mu \mathrm{m}, 0 \mu \mathrm{m})$ and blue:C $(5 \mu \mathrm{m}, 0 \mu \mathrm{m})$. The MIP corresponds to the LP$\mathrm{VM}(0.5 \%)$.

\section{Conclusions}

The FM-EDFA model based on the linearly polarized and vector modes are equivalent when the fiber core exhibits a slight ellipticity and the signals in the guided modes are incoherent. Our study show that the $D$ required for the equivalence of the two models is of the order of $0.5 \%$. This is comparable to the typical $D$ value of communication grade fiber of the order of 0.3$1.8 \%{ }^{9}$. In a MDM system, the condition of the incoherent signal modes is valid due to the absence of the cw carrier frequency and the presence of the independent phase modulation on individual signal modes.

\section{Acknowledgements}

This work was supported by the UK EPSRC grant EP/J008591/1 (COMIMO) and European Communities 7th Framework Program under grant agreement 258033 (MODE-GAP).

\section{References}

[1] Q.Kang et. al., Opt. Express 20, 20835 (2012).

[2] K.-P. Ho et. al., Opt. Express 19,16612 (2011).

[3] E. L. Lim et. al., Proc. OFC'13, OTu3G.2 (2013).

[4] D. Askarov et al, IEEE Photon. Techn. Lett., 24,1945 (2012).

[5] A. W. Snyder et. al, JOSA 68, 297 (1978).

[6] R. N. Anwar et. al., Proceedings of IEEE (ICECE) (2008).

[7] M. Born et. al., Principles of Optics, Chapter 10, Cambridge university press (1997).

[8] V.A.J.M. Sleiffer et. al. Opt. Express 20, 428 (2012).

[9] A. M. Vengsarkart al., Opt. Lett. 18,1412 (1993). 